\title{
UPPER FAITHFUL D.G. NEAR-RINGS
}

\author{
by J. D. P. MELDRUM
}

(Received 24th August 1982)

This paper is concerned with d.g. near-rings and their relationship to faithful d.g. near-rings. For general definitions and results, we refer to Pilz [5]. We use left nearrings where he uses right near-rings, but otherwise there is little difference. This work follows earlier work [3], [4] and Mahmood [2]. Before outlining the contents of the paper we present a précis of the definitions.

The set $R$ is a left zero-symmetric near-ring if it is equipped with two operations + and $\cdot$ such that $(R,+)$ is a not necessarily abelian group, $(R, \cdot)$ is a semigroup, $x(y+z)$ $=x y+x z, x 0=0 x=0$ for all $x, y, z$ in $R$. The typical such structure arises as follows. Let $(G,+)$ be a group with identity 0 and let

$$
M_{0}(G)=\{f: G \rightarrow G ; 0 f=0\}
$$

with addition defined pointwise and multiplication defined as function composition. Every (left zero-symmetric) near-ring is isomorphic to a subnear-ring of such a nearring. If $(H,+)$ is a group, then a near-ring homomorphism $\theta$ from a near-ring $R$ to $M_{0}(H)$ is a representation of $R$. As usual with ring representation theory, we omit reference to the map $\theta$ most of the time and write $h r$ for $h(r \theta)$, where $h \in H, r \in R$. We call $H$ an $R$-module. Near-ring module theory is well-developed, and the obvious terms from ring module theory carry over.

An element $s$ of $R$ is called distributive if $(x+y) s=x s+y s$ for all $x, y$ in $R$. If there exists a semigroup $S$ of distributive elements in $R$ such that $R=\mathrm{Gp}\langle S\rangle$, then $R$ is called a distributively generated (d.g.) near-ring. We write it as $(R, S)$ because the set $S$ matters. In particular $S$ need not be the semigroup of all distributive elements. In $M_{0}(G)$, End $G$, the set of all endomorphisms of $G$, is the set of all distributive elements. They generate the d.g. near-ring $(E(G)$, End $G)$. If $(R, S),(T, U)$ are two d.g. near-rings, a near-ring homomorphism $\theta: R \rightarrow T$ such that $S \theta \subseteq U$ is called a d.g. (near-ring) homomorphism. A representation $\theta: R \rightarrow M_{0}(G)$ such that $S \theta \subseteq$ End $G$ is called a d.g. representation; equivalently if $\theta$ is a d.g. homomorphism $(R, S) \rightarrow(E(G)$, End $G)$ then it is a d.g. representation. A d.g. near-ring is faithful if it has a faithful d.g. representation, that is a d.g. representation $\theta$ such that $\operatorname{Ker} \theta=\{0\}$.

Not all d.g. near-rings are faithful ([3], Theorem 3.3). But to any d.g. near-ring $(R, S)$ are associated two faithful d.g. near-rings. The lower faithful d.g. near-ring is a d.g. nearring $(\underline{R}, \underline{S})$ and a d.g. epimorphism $\theta:(R, S) \rightarrow(\underline{R}, \underline{S})$ such that for any d.g. 
homomorphism $\phi:(R, S) \rightarrow(T, U)$ where $(T, U)$ is faithful there exists a unique d.g. homomorphism $\psi:(\underline{R}, \underline{S}) \rightarrow(T, U)$ such that $\theta \psi=\phi$, ([2], Theorem 2.1). This was the main subject matter of [4]. The upper faithful d.g. near-ring is a d.g. near-ring $(\bar{R}, S)$ and a d.g. epimorphism $\theta:(\bar{R}, S) \rightarrow(R, S)$, with $\left.\theta\right|_{S}$ being the identity, such that for any d.g. homomorphism $\phi:(T, U) \rightarrow(R, S)$ where $(T, U)$ is faithful, there exists a unique d.g. homomorphism $\psi:(T, U) \rightarrow(\bar{R}, S)$ such that $\psi \theta=\phi([2]$, Theorem 2.2.).

In [4], a group-theoretic approach to finding $(\underline{R}, \underline{S})$ for a given $(R, S)$ was adopted and some examples were studied. Here we adopt the same approach for $(\bar{R}, S)$, and look at the difference between $(\bar{R}, S)$ and $(\underline{R}, \underline{S})$ for some particular cases. We measure this difference by the size of the ideal which is the kernel of the canonical homomorphism $\theta:(\bar{R}, S) \rightarrow(\underline{R}, \underline{S})$ via $(R, S)$. If $(R, S)$ is faithful this kernel is $\{0\}$, since then $(\bar{R}, S)=(R, S)$ $=(\underline{R}, \underline{S})$. If $(R, S)$ is not faithful, then $(\bar{R}, S)>(R, S)>(\underline{R}, \underline{S})$ and so it is non-zero and its size in relation to $(\widetilde{R}, S)$ will give some idea of the unfaithfulness of $(R, S)$.

\section{The upper faithful d.g. near-ring}

To develop a method for finding the upper faithful d.g. near-ring for a given d.g. nearring $(R, S)$, we need some results from earlier papers and group theory. For results from group theory we give as standard reference Scott [6]. In [3] the theory of free d.g. nearrings due to Fröhlich [1] was generalised. In any variety $\mathbf{V}$ of groups, and given any semigroup $S$, let $\operatorname{Fr}(S)$ be the free group on the set of symbols $S$. Then by defining multiplication using left distributivity, right distributivity of the elements $S$ and the product in $S$, we have a d.g. near-ring $(\operatorname{Fr}(S), S)$ which is the free d.g. near-ring on $S$ in $\mathbf{V}$, and such that given any d.g. near-ring $(R, S)$ with $(R,+)$ in $\mathbf{V}$ there is an epimorphism $\pi:(\operatorname{Fr}(S), S) \rightarrow(R, S)$ extending the identity mapping on $S$.

From group theory, we need the idea of presentations. Given a set $X$, we can define the free group $\operatorname{Fr}(X)$ on the set $X$. A set $\mathscr{R}$ of elements of $\operatorname{Fr}(X)$ generate a normal subgroup $N$. Then a group $G \cong \operatorname{Fr}(X) / N$ is said to be given by the presentation $\mathrm{Gp}\langle X ; \mathscr{R}\rangle$ where $X$ is a set of generators and $\mathscr{R}$ is a set of relations. It is fairly obvious that many different sets of relations may define the same group. In particular, given a d.g. near-ring $(R, S)$ we have a presentation for $(R,+)$ with generators $S:(R,+)$ $=\mathrm{Gp}\langle S ; \mathscr{R}\rangle$ for some set of relations $\mathscr{R}$.

Let $(R, S)$ be a non-faithful d.g. near-ring. Then $(R, S)$ is a homomorphic image of $(\operatorname{Fr}(S), S)$ and the homomorphism $\pi:(\operatorname{Fr}(S), S) \rightarrow(R, S)$ extends the identity map on $S$, and has kernel I say. Hence

$$
(R, S) \cong(\operatorname{Fr}(S), S) / \mathrm{I}
$$

The upper faithful d.g. near-ring $(\bar{R}, S)$ is also generated by $S$, so again we have

$$
(\bar{R}, S) \cong(\operatorname{Fr}(S), S) / J
$$

for some ideal $J$. Furthermore as the identity map from $S$ to $S$ extends to a d.g. homomorphism from $(\bar{R}, S) \rightarrow(R, S)$ we must have 
Since $(R, S)$ is not faithful, $S$ cannot contain a (multiplicative) identity ([3], Theorem 3.4). Let $S^{1}$ be $S$ with a multiplicative identity adjoined. So $S$ is a proper subsemigroup of $S^{1}$. We can embed $(\operatorname{Fr}(S), S)$ in $\left(\operatorname{Fr}\left(S^{1}\right), S^{1}\right)$ in a natural way and we will assume from now on that $(\operatorname{Fr}(S), S)$ is contained in $\left(\operatorname{Fr}\left(S^{1}\right), S^{1}\right)$. By Theorem 2.3 of $[4]$, since $(\bar{R}, S)$ is faithful, $(\bar{R}, S)$ can be embedded in $\left(T, S^{1}\right)$ where $(T,+)=\operatorname{Gp}\langle 1\rangle *(\bar{R},+)$ is the free product in $\mathbf{V}$ of $(\bar{R},+)$ and the free group in $\mathbf{V}$ on one symbol, namely 1 . Let $K$ be the normal closure in $\left(\operatorname{Fr}\left(S^{1}\right),+\right)$ of $(J,+)$. Then by Lemma 4.5 of [3], a standard result, we have $(T,+) \cong\left(\operatorname{Fr}\left(S^{1}\right),+\right) / K$ and so $\left(T, S^{1}\right) \cong\left(\operatorname{Fr}\left(S^{1}\right), S^{1}\right) / K$. Thus $K$ is an ideal of $\operatorname{Fr}\left(S^{1}\right)$. We also have $K \cap \operatorname{Fr}(S)=J$. By a simple application of Lemma 2.2, it follows that $\operatorname{Fr}(S)$ is a two-sided invariant subnear-ring of $\operatorname{Fr}\left(S^{1}\right): \operatorname{Fr}\left(S^{1}\right) \operatorname{Fr}(S) S^{1} \subseteq \operatorname{Fr}(S)$. It follows that

$$
\operatorname{Fr}\left(S^{1}\right) J S^{1} \subseteq J
$$

Definition 1.1. An ideal $L$ of $\operatorname{Fr}(S)$ such that $\operatorname{Fr}\left(S^{1}\right) L S^{1} \subseteq L$ is called an $\operatorname{Fr}\left(S^{1}\right)$-ideal.

Some immediate consequences are stated without proof.

Lemma 1.2. The sum of $\operatorname{Fr}\left(S^{1}\right)$ ideals is an $\operatorname{Fr}\left(S^{1}\right)$ ideal. If $L$ is an $\operatorname{Fr}\left(S^{1}\right)$ ideal of $\operatorname{Fr}(S)$, then $M$, the normal closure of $L$ in $\left(F r\left(S^{1}\right),+\right)$ is an ideal of $\operatorname{Fr}\left(S^{1}\right)$ and $M \cap \operatorname{Fr}(S)$ $=L$.

The argument above, which only depends on the fact that $(\bar{R}, S)$ is faithful, and not on the fact that it is the upper faithful d.g. near-ring for $(R, S)$, gives us the following result.

Lemma 1.3. Let $L$ be an ideal of $\operatorname{Fr}(S)$ such that $\operatorname{Fr}(S) / L$ is faithful. Then $L$ is an $\operatorname{Fr}\left(S^{1}\right)$ ideal of $\operatorname{Fr}(S)$.

This leads to our first important result.

Theorem 1.4. Let $(R, S)$ be a d.g. near-ring, I the ideal of $(\operatorname{Fr}(S), S)$ defined by $(R, S) \cong(\operatorname{Fr}(S), S) / I$. Identify $(\operatorname{Fr}(S), S)$ with its natural image in $\left(\operatorname{Fr}\left(S^{1}\right), S^{1}\right)$. Then $(\bar{R}, S)$, the upper faithful d.g. near-ring for $(R, S)$ is isomorphic to

$$
(\bar{R}, S) \cong(\operatorname{Fr}(S), S) / K
$$

where $K$ is the largest $\operatorname{Fr}\left(S^{1}\right)$ ideal of $\operatorname{Fr}(S)$ contained in $I$.

Proof. Lemma 1.2 ensures there is a unique largest $\operatorname{Fr}\left(S^{1}\right)$ ideal contained in $I$. By Lemma 1.3 and the remarks before Definition 1.1, it follows that $(\bar{R}, S)$ is isomorphic to $(\operatorname{Fr}(S), S) / K$, where $K$ is an $\operatorname{Fr}\left(S^{1}\right)$-ideal of $\operatorname{Fr}(S)$ contained in $I$.

Suppose that $I \supseteq L \supseteq K$, and $L$ is an $\operatorname{Fr}\left(S^{1}\right)$ ideal of $\operatorname{Fr}(S)$. Then by Lemma 1.3 $\operatorname{Fr}(S) / L$ is faithful. Because $L \subseteq I$, we can extend the identity map on $S$ to a d.g. homomorphism from $(\operatorname{Fr}(S), S) / L$ to $(\operatorname{Fr}(S), S) / I \cong(R, S)$. By the definition of $(\bar{R}, S)$, this can be factored through $(\tilde{R}, S) \cong(\operatorname{Fr}(S), S) / K$. This forces $K \supseteq L$. Hence $K$ is the unique largest $\operatorname{Fr}\left(S^{1}\right)$ ideal of $\operatorname{Fr}(S)$ contained in $I$.

It is also possible to prove Theorem 1.4 by a completely different route which emphasizes the presentation aspect. We use the following result from [4]. 
Theorem 1.5 Let $(R,+)=\mathrm{Gp}\langle S ; \mathscr{R}\rangle$ be a presentation of the d.g. near-ring $(R, S)$ where $\mathscr{R}=\left\{r_{\lambda} ; \lambda \in \Lambda\right\}$. Then $(R, S)$ is faithful if and only if

$$
r_{\lambda}\left(w\left(\mathbf{u} s_{1}\right), \ldots, w\left(\mathbf{u s} s_{t}\right)\right) \in H
$$

for all $\lambda \in \Lambda, w(\mathbf{u}) \in \operatorname{Fr}\left(S^{1}\right)-\operatorname{Fr}(S)$, where $H$ is the normal closure in $\operatorname{Fr}(S)$ of $\mathscr{R}$, $r_{\lambda}=r_{\lambda}\left(s_{1}, \ldots, s_{t}\right), w(\mathbf{u})=w\left(u_{1}, \ldots, u_{v}\right)$ and $w(\mathbf{u s})=w\left(u_{1} s, \ldots, u_{v} s\right)$.

This is Theorem 2.5 of [4]. Let $(R, S)$ be a d.g. near-ring with $(R, S) \cong(\operatorname{Fr}(S), S) / I$, where $I$ is the normal closure of $\mathscr{R}$ in $\operatorname{Fr}(S)$ and $(R,+)=\mathrm{Gp}\langle S ; \mathscr{R}\rangle$ is a presentation of $(R,+)$. From the proof of Theorem 2.5 in [4], it follows that an ideal $J$ of $\operatorname{Fr}(S)$ is an $\operatorname{Fr}\left(S^{1}\right)$ ideal of $\operatorname{Fr}(S)$ if and only if $r_{\lambda}\left(w\left(\mathbf{u} s_{1}\right), \ldots, w\left(\mathbf{u} s_{t}\right)\right) \in J$ for all $\lambda \in \Lambda, w(\mathbf{u}) \in \operatorname{Fr}\left(S^{1}\right)$ $-\operatorname{Fr}(S)$ where $\left\{r_{\lambda} ; \lambda \in \Lambda\right\}$ is a set which generates $J$ as a normal subgroup. So the largest $\operatorname{Fr}\left(S^{1}\right)$ ideal contained in $I$, identifying $(\operatorname{Fr}(S), S)$ with its natural image in $\left(\operatorname{Fr}\left(S^{1}\right), S^{1}\right)$, is the largest set $\mathscr{R}_{1}$ satisfying the condition of Theorem 1.5 , which is contained in $I$. Since $(\bar{R}, S)$ is mapped onto $(R, S)$ by a d.g. homomorphism which extends the identity map on $S$, the kernel $L$ of the canonical homomorphism $(\operatorname{Fr}(S), S) \rightarrow(\bar{R}, S)$ is contained in $I$, i.e. $L \subseteq I$. But $L$ is an $\operatorname{Fr}\left(S^{1}\right)$ ideal of $\operatorname{Fr}(S)$, so $L \subseteq K$. Also $\operatorname{Fr}(S) / K$ is faithful by Theorem 1.3. So $(\tilde{R}, S)$ is a homomorphic image of $\operatorname{Fr}(S) / K$ and $K \subseteq L$. Thus $K=L$ and we have another proof of Theorem 1.4 .

\section{Some applications}

In [4], the lower faithful d.g. near-rings were calculated for some d.g. near-rings of a particularly simple kind. We will consider some of these d.g. near-rings here and find their upper faithful d.g. near-rings. In practice, the method we use for finding a presentation for the upper faithful d.g. near-ring is based on the original proof of existence in [3].

Consider the d.g. near-ring $(R, S)$. Construct the group $G=\mathrm{Gp}\langle x\rangle *\left(R_{x},+\right)$, the free product of an infinite cyclic group generated by $x$ and a copy of $(R,+)$ which we denote $R_{x}=\left\{r_{x} ; r \in R\right\}$. Define $\bar{S}=\{\bar{s} ; s \in S\}$ as a semigroup of endomorphisms of $G$ by

$$
x \bar{s}=s_{x}, r_{x} \bar{s}=(r s)_{x}
$$

where the product in $(r s)_{x}$ is the product obtained from $R$ and $\bar{s}$ is the endomorphism induced by $s \in S$. Then the d.g. near-ring generated by $\bar{S}$ in $M_{0}(G)$ is $(\bar{R}, \bar{S})$ ([3], Theorem 4.6). Let $\varepsilon_{1} \bar{s}_{1}+\ldots+\varepsilon_{n} \bar{s}_{n}$ be a word in the elements of $\bar{S}$, where $\varepsilon_{i}= \pm 1$ for $1 \leqq i \leqq n$. An arbitrary element $g$ of $G$ is

$$
g=m_{1} x+r_{1 x}+m_{2} x+r_{2 x}+\ldots+m_{t} x+r_{t x}
$$

where $m_{j}$ is an integer, $m_{1}$ may be $0, r_{t x}$ may be $0_{x}$ but all other terms are non-trivial. Then the word $\varepsilon_{1} \bar{s}_{1}+\ldots+\varepsilon_{n} \bar{s}_{n}$ is a relation in $\bar{R}$ if and only if

$$
g\left(\varepsilon_{1} \bar{s}_{1}+\ldots+\varepsilon_{n} \bar{s}_{n}\right)=0
$$


for all $g \in G$, that is if and only if

$$
\left(m_{1} x+r_{1 x}+\ldots+m_{t} x+r_{t x}\right)\left(\varepsilon_{1} \bar{s}_{1}+\ldots+\varepsilon_{n} \bar{s}_{n}\right)=0
$$

for all $t \geqq 1, m_{i} \in Z, r_{i} \in R$. We consider this product more closely:

$$
\begin{aligned}
\left(m_{1} x\right. & \left.+r_{1 x}+\ldots+m_{t} x+r_{t x}\right)\left(\varepsilon_{1} \bar{s}_{1}+\ldots+\varepsilon_{n} \bar{s}_{n}\right) \\
= & \varepsilon_{1}\left(m_{1} x+r_{1 x}+\ldots+m_{t} x+r_{t x}\right) \bar{s}_{1}+\ldots+\varepsilon_{n}\left(m_{1} x+r_{1 x}+\ldots+m_{t} x+r_{t x}\right) \bar{s}_{n} \\
= & \varepsilon_{1}\left(m_{1} s_{1 x}+\left(r_{1} s_{1}\right)_{x}+\ldots+m_{t} s_{1 x}+\left(r_{t} s_{1}\right)_{x}\right)+\ldots+\ldots \\
& +\ldots+\varepsilon_{n}\left(m_{1} s_{n x}+\left(r_{1} s_{n}\right)_{x}+\ldots+m_{t} s_{n x}+\left(r_{t} s_{n}\right)_{x}\right)
\end{aligned}
$$

We can now state the general result concerning presentations of upper faithful d.g. nearrings.

Theorem 2.2. Let $(R, S)$ be a d.g. near-ring. With the notation given just above, a presentation for the upper faithful d.g. near-ring $(\bar{R}, \bar{S})$ for $(R, S)$ is given by

$$
\operatorname{Gp}\left\langle\bar{S} ; \varepsilon_{1} \bar{s}_{1}+\ldots+\varepsilon_{n} \bar{s}_{n} \in \mathscr{R}_{1}\right\rangle
$$

where $\mathscr{R}_{1}$ is the set of all words $\varepsilon_{1} \bar{s}_{1}+\ldots+\varepsilon_{n} \bar{s}_{n}$ such that $(2.1)$ is zero in $R$ for all choices of $t \geqq 1, m_{i} \in Z, r_{i} \in R, m_{i} \neq 0$ for $i \geqq 2, r_{i} \neq 0$ for $i \leqq t-1$.

This result is theoretical, but not practical in general and compares with Theorem 2.6 of [4]. But in special cases it can produce some concrete results. As in [4], there is a class of near-rings which yields much easier presentations, namely the class of zero nearrings in which all products are zero. They are obviously d.g. near-rings since all elements are distribative. In this case Theorem 2.2 takes a much simpler form.

Theorem 2.3. Let $(R, S)$ be a zero d.g. near-ring. Then the upper faithful d.g. near-ring $(\bar{R}, \bar{S})$ for $(R, S)$ is given by

$$
\operatorname{Gp}\left\langle\bar{S} ; \mathscr{R}_{1}\right\rangle
$$

the zero d.g. near-ring, where $\mathscr{R}_{1}$ is the set

$$
\left\{\varepsilon_{1} \bar{s}_{1}+\ldots+\varepsilon_{n} \bar{s}_{n} ; \varepsilon_{1} m s_{1}+\ldots+\varepsilon_{n} m s_{n}=0 \text { in } R \text { for all } m \in Z\right\} .
$$

Proof. This follows immediately because the right hand side of (2.1) becomes, in this situation

$$
\varepsilon_{1}\left(\left(\sum_{j=1}^{t} m_{j}\right) s_{1 x}\right)+\ldots+\varepsilon_{n}\left(\left(\sum_{j=1}^{t} m_{j}\right) s_{n x}\right)
$$


Compare this with Lemma 3.3 of [4]. In the notation of that paper we are just taking the maximal integrally closed set of relations contained in the relations for $R$. We now apply this to the zero d.g. near-rings on the dihedral groups. These groups were studied in [4] and their lower faithful d.g. near-rings were determined.

We define the dihedral groups $D_{2 n}$ of order $2 n$ by the presentation

$$
D_{2 n}=\mathrm{Gp}\langle x, y ; 2 x, n y, x+y+x+y) .
$$

This group is often denoted $D_{n}$, but we will use the same notation as in [4]. Normally we restrict $n$ to be at least 3 . The detailed structure of the group $D_{2 n}$ is well-known and can be obtained from most textbooks on group theory. We will mention the results we need without proof when appropriate. We take as our set of distributive generators the set of elements $\{x, y\}$. To simplify our notation we will write the two generators of the upper faithful d.g. near-ring as $\{s, t\}, s$ corresponding to $x, t$ to $y$ and the near-ring itself as $(R, S)$. It will of course itself be a zero d.g. near-ring. So all we need to do is to determine its additive structure using Theorem 2.3.

Lemma 2.4. The complete set of relations for $(R, S)$ is

$$
\left\{2 s, n t, p_{1} t+s+p_{2} t+\ldots+s+p_{2 m+1} t\right\}
$$

for all $p_{1}, \ldots, p_{2 m+1}$ such that

$$
\begin{gathered}
2 \sum_{i=1}^{2 m+1} p_{i} \equiv 0 \bmod n, \\
\sum_{i=1}^{2 m+1}(-1)^{i} p_{i} \equiv 0 \bmod n .
\end{gathered}
$$

Proof. We first show that these relations hold. The first two follow since $2 m x, n m y$ are relations which hold in $D_{2 n}$ for all $m \in Z$. So consider

$$
p_{1} t+s+p_{2} t+\ldots+s+p_{2 m+1} t
$$

We need to show that for all $q \in Z$,

$$
p_{1} q y+q x+p_{2} q y+\ldots+q x+p_{2 m+1} q y=0
$$

in $D_{2 n}$ for all $q \in Z$. If $q$ is even then the left hand side becomes $q\left(\sum_{i=1}^{2 m+1} p_{i}\right) y$, and this is zero when $2 \sum_{i=1}^{2 m+1} p_{i} \equiv 0 \bmod n$. If $q$ is odd, then the left hand side becomes

$$
\begin{aligned}
& p_{1} q y+x+p_{2} q y+\ldots+x+p_{2 m+1} q y \\
& \quad=2 m x+p_{1} q y-p_{2} q y+\ldots+p_{2 m+1} q y \\
& =-q\left(\sum_{i=1}^{2 m+1}(-1)^{i} p_{i}\right) y .
\end{aligned}
$$


since $x+r y=-r y+x$ in $D_{2 n}$. So all these relations do hold in $R$.

Conversely let

$$
p_{1} t+s+p_{2} t+\ldots+s+p_{m} t
$$

be a relation in $R$ where $p_{1}$ and $p_{m}$ may be 0 . Then $p_{1} y+x+\ldots+x+p_{m} y=0$ in $R$ and so

$$
(m-1) x+(-1)^{m-1} p_{1} y+(-1)^{m-2} p_{2} y+\ldots+p_{m} y=0 .
$$

So we must have $m-1$ even and $\sum_{i=1}^{m}(-1)^{i} p_{i} \equiv 0 \bmod n$. Also $p_{1} 2 y+2 x+\ldots+2 x+p_{m} 2 y$ $=0$ in $R$ forces $2 \sum_{i=1}^{m} p_{i} \equiv 0 \bmod n$. This shows that the relations in the statement are a complete set of relations for $(R,+)$.

These relations are in fact greatly redundant as we shall soon see. We remark first that we can say a lot about $(R, S)$ without detailed presentations. From [3] we know that any d.g. near-ring $(T, U)$ such that $(T,+)$ is a free group in some variety on the set of generators $U$ is faithful. As $D_{2 n}$ is soluble of class 2 , then so is $(R,+)$ since this is a variety. Also $D_{2 n}$ is of exponent $n$ if $n$ is even, $2 n$ if $n$ is odd. Thus $(R,+)$ also satisfies this. Let $R^{\prime}$ be the derived group of $R$. Then $R^{\prime}$ is abelian and is generated by $[s, t]=-s$ $-t+s+t=s-t+s+t$, and all its conjugates. Since $R=\mathrm{Gp}\langle s, t\rangle, 2 s=0=n t$, it follows that $\left\{[s, t]^{a s+b t} ; a=0,1,0 \leqq b<n\right\}$ is the complete set of conjugates of $[s, t]$ (notation: $u^{v}$ $=-v+u+v$ ). In $D_{2 n}$, the derived group is generated by $[x, y]=2 y$, which has order $n$ if $n$ is odd, $n / 2$ if $n$ is even. We now determine the structure of $R$. As the two conditions on a word in $s$ and $t$ given to Lemma 2.4 will keep occurring, we will refer to them as the first and second conditions.

Lemma 2.5. $[s, t]$ has order $n$ if $n$ is odd and $n / 2$ if $n$ is even.

Proof. Consider $a[s, t]=a(-s-t+s+t)$. The first condition is automatically satisfied. The second condition gives $-2 a \equiv 0 \bmod n$. This gives the result, since $n$ odd forces $a=n, n$ even forces $a=n / 2$ as the least value satisfying the second condition.

Lemma 2.6. $[s, t]^{s}=-[s, t],[s, t]^{t}=[s, t]$.

Proof. $[s, t]^{s}=-s-s-t+s+t+s=-t-\dot{s}+t+s=[t, s]=-[s, t]$ since $2 s=0$, which is the first equation. Now consider

$$
-[s, t]+[s, t]^{t}=-t-s+t+s-t-s-t+s+t+t .
$$

This word obviously satisfies the first condition as there are an equal number of $+t$ and $-t$. The second condition is satisfied as can easily be checked. Hence $-[s, t]+[s, t]^{t}$ is a relation in $R$. This completes the proof of Lemma 2.3 .

Theorem 2.7. (i) If $n$ is odd, then

$$
(R,+)=\{a s+b t+c[s, t] ; 0 \leqq a \leqq 1,0 \leqq b, c \leqq n-1\}
$$


has order $2 n^{2}$, and a set of relations for the generators

$$
\{s, t\} \quad \text { is } \quad\left\{2 s, n t, n[s, t],[s, t]^{s}+[s, t],[s, t]^{t}-[s, t]\right\} .
$$

(ii) If $n=2 m$ and $m$ is odd, then

$$
(R,+)=\{a s+b t+c[s, t] ; 0 \leqq a \leqq 1,0 \leqq b, 2 c \leqq n-1\}
$$

has order $n^{2}$, and a set of relations for the generators

$$
\{s, t\} \quad \text { is } \quad\left\{2 s, n t, m[s, t],[s, t]^{s}+[s, t],[s, t]^{t}-[s, t]\right\} .
$$

(iii) If $n=4 m$ then

$$
(R,+)=\{a s+b t+c[s, t] ; 0 \leqq a \leqq 1,0 \leqq b, 4 c \leqq n-1\}
$$

has order $n^{2} / 2$, and a set of relations for the generators

$$
\{s, t\} \quad \text { is } \quad\left\{2 s, n t, 2 m t+m[s, t],[s, t]^{s}+[s, t],[s, t]^{t}-[s, t]\right\} .
$$

Proof. Lemmas 2.5 and 2.6, together with the general remarks concerning the general structure enable us to state the following conclusions. The group $R / R^{\prime}$ has order $2 n$, generated by $s$ of order $2, t$ of order $n, R^{\prime}=\mathrm{Gp}\langle[s, t]\rangle$ is a cyclic normal subgroup of order $n$ if $n$ is odd, $n / 2$ if $n$ is even. Lemma 2.6 gives the action of $s$ and $t$ on $R^{\prime}$. The only question that remains is whether there is a non-trivial relation of the form

$$
a s+b t+c[s, t]=a s+b t+c(-s-t+s+t)
$$

Since $x+b y+c(-x-y+x+y) \neq 0$ in $D_{2 n}$, it follows that $a$ must be 0 . So we have

$$
b t+c(-s-t+s+t)
$$

The two conditions of Lemma 2.4 give us

$$
\begin{aligned}
2 b & \equiv 0 \bmod n, \\
b+2 c & \equiv 0 \bmod n
\end{aligned}
$$

and we may obviously assume that $0 \leqq b, c \leqq n-1$.

(i) If $n$ is odd, we get in succession $b=0, c=0$ and so part (i) holds.

(ii) Let $n=2 m$, with $m$ odd. Then we can have $b=0$ or $m$ to satisfy the first congruence. From the second congruence $b=0$ forces $c=0$ or $m$, both cases being covered by earlier relations. Also $b=m$ gives $m+2 c \equiv 0 \bmod 2 m$. So $m$ divides $2 c$, and $m$ odd forces $m$ divides $c$, which gives a contradiction. Thus no further non-trivial relations exist in this case and part (ii) holds.

(iii) Let $n=4 m$. Then $b=0$ or $b=2 m$ satisfies the first congruence. The second congruence forces $c=2 m$ or 0 if $b=0$, giving no further non-trivial relations. So assume 
that $b=2 m$. Then we need $2 m+2 c \equiv 0 \bmod 4 m$. So $c=m$ is a solution. This completes the proof of part (iii).

Corollary 2.8. The zero d.g. near-ring on $D_{8}$ is faithful.

Proof. Theorem 2.7 (iii) with $m=1$ gives the result

This result is given in [4], Theorem 3.4 also. Note that the three cases are the same as were obtained in Theorem 3.4 [4] in a different context. We now introduce a measure of the lack of faithfulness of a d.g. near-ring.

Definition 2.9. Let $(R, S)$ be a d.g. near-ring with upper faithful d.g. near-ring $\theta:(\bar{R}, S) \rightarrow(R, S)$, and lower faithful d.g. near-ring $\phi:(R, S) \rightarrow(R, \underline{S})$. Then we call $\operatorname{Ker} \theta$ the upper defect of $(R, S), \bar{D}(R, S), \operatorname{Ker} \phi$ the lower defect of $(R, S), \underline{D}(R, S)$ and $\operatorname{Ker} \theta \phi$ the defect of $(R, S), D(R, S)$.

The following facts are immediate consequences of the definition.

Lemma 2.10. If either the upper defect or the lower defect of a d.g. near-ring is $\{0\}$, so is the other. The defect of a d.g. near-ring is an extension of the lower defect by the upper defect.

We can now determine easily the defects of the zero d.g. near-rings on the dihedral groups.

Theorem 2.11. Let $(R, S)$ be the zero d.g. near-ring on $D_{2 n}$ where $(R,+)$ $=\mathrm{Gp}\langle s, t ; 2 s, n t, s+t+s+t\rangle$, and $n \geqq 3$.

(i) If $n$ is odd, then $\bar{D}(R, S)$ is the zero near-ring on a cyclic group of order $n, D(R, S)$ is the zero near-ring on a cyclic group of order $n$, and $D(R, S)$ is the zero near-ring on the direct sum of two cyclic groups of order $n$.

(ii) If $n=2 m$ and $m$ is odd, then $\bar{D}(R, S)$ is the zero near-ring on a cyclic group of order $m, D(R, S)$ is the zero near-ring on a cyclic group of order $m$, and $D(R, S)$ is the zero nearring on the direct sum of two cyclic groups of order $m$.

(iii) If $n=4 m$, then $\bar{D}(R, S)$ is the zero near-ring on a cyclic group of order $m, D(R, S)$ is the zero near-ring on a cyclic group of order $m$, and $D(R, S)$ is the zero near-ring on the direct sum of two cyclic groups of order $m$.

Proof. These results follow from Theorem 2.7, Theorem 3.4 of [4] and some elementary abelian group theory. Note that in $(\bar{R}, S)$, the group generated by $t$ and $[s, t]$ is always abelian. We give the generators in each case.

(i) $D(R, S)=\mathrm{Gp}\langle t,[s, t]\rangle, \bar{D}(R, S)=\mathrm{Gp}\langle[s, t]\rangle, \underline{D}(R, S)=\mathrm{Gp}\langle t\rangle$.

(ii) $D(R, S)=\mathrm{Gp}\langle 2 t,[s, t]\rangle, \bar{D}(R, S)=\mathrm{Gp}\langle[s, t]\rangle, \underline{D}(R, S)=\mathrm{Gp}\langle 2 t\rangle$.

(iii) $D(R, S)=\mathrm{Gp}\langle 4 t,[s, t]-2 t\rangle, \bar{D}(R, S)=G p\langle[s, t]-2 t\rangle, \underline{D}(R, S)=\mathrm{Gp}\langle 4 t)$. 


\section{REFERENCES}

1. A. Fröhlich, On groups over a d.g. near-ring (I): Sum constructions and free $R$-groups, Quart. J. Math. (Oxford) (2) 11 (1960), 193-210.

2. S. J. Mahmood, Limits and colimits in categories of d.g. near-rings, Proc. Edinburgh Math. Soc. 23 (1980), 1-7.

3. J. D. P. Meldrum, The representation of d.g. near-rings, J. Austral. Math. Soc. 16 (1973), 467-480.

4. J. D. P. Meldrum, Presentations of faithful d.g. near-rings, Proc. Edinburgh Math. Soc. 23 (1980), 49-56.

5. G. Pilz, Near-rings (North-Holland, Amsterdam, 1976).

6. W. R. Scort, Group Theory (Prentice-Hall, Englewood Cliffs, N.J., 1964).

Department of Mathematics

UNIVERSITY OF EDINBURGH

King's BUILDINGS

Mayfield Road

EDINBURGH EH9 3JZ 